\title{
АДАПТАЦИЯ И ВНУТРЕННЯЯ КАРТИНА ДЕФЕКТА ВЗРОСЛЫХ С ОГРАНИЧЕННЫМИ ВОЗМОЖНОСТЯМИ ЗДОРОВЬЯ
}

\author{
Работа выполнена при финансовой поддержке Российского фонда \\ фундаментальных исследований \\ (проект № 19-013-00768A)
}

Адеева Т.Н., Тихонова И.В. (Костромской государственный университет, Кострома, Россия) adeeva.tanya@rambler.ru,inn.007@mail.ru 乙tinцшјшgर्uाu uर्u. 21.07.2021 9pmpunuर्जuध uर्णu. 09.08.2021

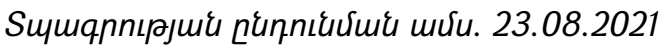

Внутренняя картина дефекта рассматривается как составляющая Яконцепции и понимается как комплекс ощущений, знаний, переживаний, мотивационных изменений, связанных с расстройством. Данный феномен включает четыре компонента: физический, когнитивный, эмоциональный, мотивационный. Особенности внутренней картины дефекта взрослых с ограниченными возможностями здоровья (ВКД) определены в исследовании. Феномен ВКД рассматривается как фактор формирования Я-концепции, а значит, может быть связан с отношением человека к себе, к окружающим, с мотивами деятельности, с формированием различных черт личности. Компоненты ВКД имеют разное содержание и выраженность в зависимости от варианта дизонтогенеза. Наибольшая выраженность компонентов ВКД наблюдается в группах взрослых людей с нарушениями функций опорно-двигательного аппарата и нарушениями слуха. Внутренняя картина дефекта имеет значение для социальнопсихологической адаптации личности. В исследовании определена связь ВКД с такими показателями адаптации как эмоциональный комфорт, внешний и внутренний контроль, доминирование, уход от проблем. Некоторые из параметров ВКД являются предикторами параметров адаптации при различных вариантах нарушенного развития.

Ключевые слова: внутренний паттерн расстройства, дизонтогенез, адаптация, нарушения слуха, нарушения зрения, нарушения функций опорнодвигательного аппарата.

DOI: https://doi.org/10.46991/SBMP/2021.4.2.003

В данной работе внимание обращено к двум аспектам, связанным с развитием и фрункционированием личности. Ограниченные возможности здоровья часто рассматриваются как условия, связанные с трудностями 
социально-психологической адаптации и самореализации личности. Проблема адаптации рассматривается в современной психологии в нескольких аспектах. Большое внимание уделяется вопросам адаптации личности в образовательном пространстве и в профессиональной сфере. С одной стороны, обосновывается необходимость грамотного психолого-педагогического сопровождения профессионального становления лиц с ОВ3 [3; 6]. С другой стороны, ситуация развития личности рассматривается как фактор, способствующий направленности человека на сотрудничество и помощь. В других работах подчеркивается значимость социальных влияний. В первую очередь, внимание обращено к феномену стигмации, который связывается с возможными проявлениями депрессии, негативной самооценки, низкой социальной активности личности. В отечественных и зарубежных источниках определено опосредованное влияние данного френомена на проявление негативных черт личности [1, 5, 9]. Другим значимым социальным фрактором является наличие организованной социальной поддержки и проблема одиночества людей с ограниченными возможностями здоровья [8]. Наконец, ряд работ посвящен определению личностных, индивидуальных факторов, важных для процесса адаптации. Изучаются личностные характеристики, варианты совладающего поведения, особенности самосознания лиц с ОВЗ. На первый план выходят проблемы формирования позитивного самоотношения, адекватной самооценки, формирования системы социальных связей [2, 4, 7].

Исследования внутренней картины дефекта (ВКД) позволяют увидеть феноменологию фракторов, значимых для социально-психологической адаптации, а, кроме того, возможно, и описать механизм возникновения рисков дезадаптации. Внутренняя картина дефект понимается в нашем исследовании как сложное, интегративное образование, как комплекс ощущений, знаний, переживаний, мотивационных изменений, связанных с нарушением. Сенситивный компонент включает в себя совокупность ощущений, связанных с наличием дефекта. Физический компонент связан с характеристикой человеком собственной фризической активности, ффизических качеств. Когнитивный компонент представляет собой знания о причинах, проявлениях дефекта, ограничениях, связанных с его наличием. Эмоциональный компонент означает отношение личности к дефекту. Мотивационный компонент, характеризует структуру мотивов личности, возможное ее изменение в связи с наличием дефекта. Внутренняя картина дефекта рассматривается как составляющая Яконцепции, она может быть связана с особенностями адаптации личности.

Цель исследования - выявление взаимосвязей между наполненностью компонентов внутренней картины дефекта и параметрами адаптации у взрослых с различными вариантами дизонтогенеза. 
В исследовании принимали участие 75 взрослых с ограниченными возможностями здоровья. Представлена целенаправленная выборка, все респонденты имеют нарушения развития с детства. Их них: 21 человек с нарушениями зрения (Н3, 7 мужчин, 14 женщин), 19 человек с тяжелыми нарушениями речи (THP, 10 мужчин, 9 женщин), 24 человека с нарушениями функций опорно - двигательного аппарата (ОДА, 14 мужчин, 10 женщин), 11 человек с нарушениями слуха (НC, 5 мужчин, 6 женщин). Средний возраст 35 лет. В исследовании применялся опросный метод и метод беседы. Исследование проводилось индивидуально.

Методы исследования. Использована беседа «Изучение внутренней картины дефекта» Т.Н. Адеевой (2018). Разделы беседы были структурированы в соответствии с выделенными компонентами ВКД (сенситивный, физический, когнитивный, эмоциональный, мотивационный). Полученные ответы респондентов были обработаны методом контент-анализа. Использованы «Методика диагностики социально-психологической адаптации К. Роджерса и Р. Даймонда» (адаптация А.К. Осницкого, 2004), Статистическая обработка проводилась с использованием программы 10.0 Statistica, для выявления взаимосвязи наполненности компонентов ВКД и параметров адаптации вычислялся коэффрициент корреляции r-Спирмена; для выявления детерминации компонентов ВКД применялся регрессионный анализ.

Результаты исследования. Компоненты внутренней картины дефекта имеют разную наполненность в группах респондентов С различными вариантами дизонтогенеза (табл. 1). В группе людей с нарушениями функций опорно-двигательного аппарата (ОДА) и нарушениям зрения (Н3) отмечена большая наполненность физического компонента по сравнению с другими группами. При нарушении функций ОДА респонденты указывают на наличие боли, дискомфорта, утомляемости, когда выполняют разные виды деятельности. Респонденты с нарушениями зрения отмечают наличие разнообразных, часто труднодиффреренцируемых и пугающих ощущений. 
Таблица 1

Наполвенность компонентов ВКД у взросльхх с различнымих вариантами дизонтогенеза

\begin{tabular}{|l|l|l|l|l|}
\hline $\begin{array}{l}\text { Наполненность компонентов } \\
\text { Варианты дизонтогенеза }\end{array}$ & $\begin{array}{l}\text { Нарушения } \\
\text { зрения }\end{array}$ & $\begin{array}{l}\text { Нарушения } \\
\text { речи }\end{array}$ & $\begin{array}{l}\text { Нарушения } \\
\text { слуха }\end{array}$ & $\begin{array}{l}\text { Нарушение } \\
\text { функций } \\
\text { ОДА }\end{array}$ \\
\cline { 1 - 4 } Компоненты ВКД & 4,42 & 2,84 & 2,63 & 4,33 \\
\hline физический & 4,1 & 4,8 & 6,0 & 5,8 \\
\hline когнитивный & 3,68 & 4,1 & 6,09 & 6,0 \\
\hline эмоциональный & 2,53 & 4,6 & 5,55 & 5,3 \\
\hline мотивационный & \multicolumn{3}{|l}{} \\
\hline
\end{tabular}

В группах людей с нарушениями слуха и нарушениями функций ОДА присутствует достаточная информированность о нарушении (когнитивный компонент), эмоциональное переживание, отношение к состоянию (эмоциональный компонент), особенности мотивации в связи с наличием нарушения.

Изучение корреляционных связей показало, что во всей выборке присутствуют значимые корреляционные связи между фризическим компонентом и компонентами адаптации. Выраженность фризического компонента связана с эмоциональным дискомфортом $(r=0,32, p<0,05)$, дезадаптацией $(r=0,27, p<0,05)$, внешним контролем $(r=0,33, p<0,05)$, со снижением интернальности $(r=0,29, p<0,05)$. В группе людей с Н3 и тяжелыми нарушениями речи (ТHР) корреляционные связи зафиксированы только между параметрами адаптации и физическим компонентом. У респондентов с Н3 физический компонент связан с снижением адаптации $(r=-0,46, p<0,05)$, принятием себя $(r=0,46, p<0,05)$. В группе людей с ТНР физический компонент связан с дезадаптацией $(r=0,58, p<0,05)$, лживостью $(r=0,4, p<0,05)$, неприятияем себя $(r=0,58, p<0,05)$, эмоциональным дискомфортом $(r=0,68$, $p<0,05)$, внешним контролем $(r=0,75, p<0,05)$. В группе людей с $\mathrm{HC}$ физический компонент связан с ведомостью $(r=0,63, p<0,05)$ и снижением стремления к доминированию $(r=-0,61, p<0,05)$. Можно предположить, что внимание к физическим проявлениям нарушения связано с проблемами адаптации. Особенно значимо это для группы людей с НС, поскольку выраженность физического компонента здесь довольно высокая. Интересно, но в группе людей с нарушениями функций ОДА фризический компонент не имеет связи с параметрами адаптации. На первый взгляд, это может говорить о достаточном уровне самопонимания и самопринятия, однако требуется более 
глубокий анализ, поскольку регрессионный анализ показывает не столь однозначные результаты.

В группах людей с нарушениями функций ОДА и НС присутствуют корреляционные связи когнитивного, эмоционального и мотивационного компонентов с параметрами адаптации. В группе лиц с нарушениями функций ОДА осведомленность о своем нарушении связана с адаптивностью $(r=0,5, p$ $<0,05)$, эмоциональным контролем $(r=0,58, p<0,05)$, внутренним контролем $(r=0,44, p<0,05)$. Однако в то же время отмечена связь с параметром лживости $(r=0,47, p<0,05)$, что может говорить о действии защитных механизмов. При проведении содержательного анализа ответов респондентов было отмечено, что знания о причинах имеющегося нарушения достаточно стереотипны, в основном, люди говорят об «травмах во время родов», о «врожденной проблеме». Достаточно полное, адекватное представление о причинах нарушения имеют немногие. Стремление к преодолению ограничений в данной группе связано с эмоциональным комфрортом ( $r=0,45$, p <0,05), а эмоциональный компонент связан с принятием других $(r=0,43, p<0,05)$. Предполагаем, что возможность достаточно открыто говорить о своих проблемах и выражать отношение к ним позволяет снизить дистанцию между людьми.

В группе людей с НС знания о дефекте связаны с принятием других $(r=0,64, p<0,05)$, знания о дефекте и возможность выразить отношение к состоянию связаны с внутренним контролем $(r=0,79, p<0,05 ; r=0,68, p<0,05)$. Изменение мотивации с учетом нарушения связано с принятием других $(r=0,64$, $p<0,000)$, со снижением лживости $(r=-0,76, p<0,05)$, однако снижается и эмоциональный комфорт $(r=-0,74, p<0,05)$. Скорее всего, это говорит о высокой затратности усилий по адаптации и, возможно, недостаточности социальных и личностных ресурсов.

На следующем этапе исследования было определено влияние компонентов ВКД на ряд параметров адаптации. Для всей выборки респондентов имеет значение физический компонент. Он оказывает негативное влияние $(\beta=-0,233$, $\mathrm{p}=0,046)$ на параметр адаптации $\left(\mathrm{R}^{2}=0,054 ; \mathrm{F}=4,115 ; \mathrm{p}=0,046\right)$, а также снижает $(\beta=-0,281, p=0,015)$ интернальность $\left(R^{2}=0,079 ; F=6,156 ; p=0,015\right)$, является предиктором ( $\left.R^{2}=0,079 \mathrm{~F}=6,16 ; \mathrm{p}=0,015\right)$, снижающим эмоциональный комфорт $\left(\mathrm{R}^{2}=0,079 \mathrm{~F}=6,16 ; \mathrm{p}=0,015\right)$.

В группе респондентов с ТНР фризический компонент также является единственным из компонентов, влияющим на параметры адаптации.

В группе людей с НС когнитивный компонент оказывает влияние $(\beta=0,615 ; p=0,044)$ на интернальность $\left(R^{2}=0,378 ; F=5,480 ; p=0,044\right)$, внимание к физическим ощущениям снижает $(\beta=-0,679 p=0,022)$ стремление к доминированию $\left(R^{2}=0,461 F=7,698 ; p=0,022\right)$. 
Таблица 2

Детерминапия параметров адаптапии в групше респондентов с ТHP

\begin{tabular}{|c|c|c|c|c|c|}
\hline \multicolumn{2}{|c|}{ Компоненты ВКД } & Физический & Физический & Физический & Физический \\
\hline \multicolumn{2}{|c|}{$\begin{array}{l}\text { Показатели значимости } \\
\text { регрессионной модели }\end{array}$} & $\begin{array}{l}\mathrm{R} 2=0,287 \mathrm{~F} \\
=6,852 ; \\
\mathrm{p}=0,018\end{array}$ & $\begin{array}{l}\mathrm{R} 2=0,452 \mathrm{~F}= \\
14,019 ; \\
\mathrm{p}=0,002\end{array}$ & $\begin{array}{l}\mathrm{R} 2=0,316 \mathrm{~F}= \\
7,848 ; \\
\mathrm{p}=0,012\end{array}$ & $\begin{array}{l}\text { R2=0,313 F } \\
=7,741 \\
p=0,013\end{array}$ \\
\hline \multirow{4}{*}{ 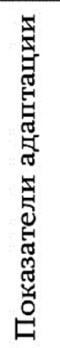 } & Адаптация & $\begin{array}{l}\beta=-0,536 \\
p=0,018\end{array}$ & & & \\
\hline & Интернальность & & $\begin{array}{l}\beta=-0,672 \\
p=0,002\end{array}$ & & \\
\hline & Самопринятие & & & $\begin{array}{l}\beta=-0,562 \\
p=0,002\end{array}$ & \\
\hline & $\begin{array}{l}\text { Эмоциональная } \\
\text { комфортность }\end{array}$ & & & & $\begin{array}{l}\beta=-0,559 \\
p=0,013\end{array}$ \\
\hline
\end{tabular}

В группах людей с Н3 и нарушениями функций ОДА не выявлено влияния компонентов ВКД на параметры адаптации. Однако компоненты внутренней картины дефекта в данных группах являются предикторами для некоторых параметров самоотношения и индивидуально-личностных характеристик. Эти показатели, в свою очередь, оказываются связанными с параметрами адаптации, поэтому можем предположить, что влияние ВКД в группах людей с НЗ и нарушениями функций ОДА является опосредованным и требует детального анализа.

Выводы. Наполненность компонентов внутренней картины дефекта отличается в группах взрослых с различными вариантами дизонтогенеза.

Наибольшее количество корреляционных связей обнаружено между параметрами адаптации и ффизическим компонентов ВКД. Физический компонент ВКД выступает предиктором некоторых параметров адаптации в группе людей с ТНР и НС. Когнитивный компонент определяет повышение внутреннего контроля в группе людей с нарушениями слуха.

Предполагаем, что влияние компонентов ВКД на параметры адаптации в группах людей с нарушениями зрения и нарушениями функций ОДА является опосредованным и требует детального анализа.

\section{Литература}

1. Бондаренко Г.Л. Я - концепция людей с инвалидностью // Дефектология. 2006, №5, с. $36-40$.

2. Горынина В.С. Психологические особенности лиц с нарушениями зрения с высоким уровнем социальной активности // Социализация и 
реабилитация в современном мире. Сборник научных статей. Под редакцией О.Е. Нестеровой, Р.М. Шамионова, Л.В. Шиповой, Е.С. Пяткиной, М.Д. Коноваловой. Москва, 2017 Издательство: Издательство "Перо" (Москва), с. 544 - 558.

3. Пряжникова Е. Ю., Сергеева М. Г. Психологические особенности профессионального самосознания студентов с ограниченными возможностями здоровья//Вестник московского института лингвистики. № 1, 2015, c. 96 - 105.

4. Чебарыкова С.В. Личность в условиях дизонтогенеза: внутренняя картина дефекта. Хабаровск: Изд-во Тихоокеан. гос. ун-та, 2017.

5. Bae S. , Lee. S., Harada K., Makizako H., Park H., Shimada H. Combined effect of self-reported hearing problems and level of social activities on the risk of disability in Japanese older adults: A populationbased longitudinal study. Maturitas. 2018. Vol.115, pp. 51-55. https://doi.org/10.1016/j.maturitas.2018.06.008

6. Bonaccio, S., Connelly, C.E., Gellatly, I.R. et al. The Participation of People with Disabilities in the Workplace Across the Employment Cycle: Employer Concerns and Research Evidence // J Bus Psychol. 2020. Vol. 35, pp. 135-158. https://doi.org/10.1007/s10869-018-9602-5.

7. Dirk-Wouter S., Van Gorp M., Van Wely,L., Verheijden J., Voorman J., Wintels S., Van der Cruijsen J., Ketelaar K. Participation in Social Roles of Adolescents With Cerebral Palsy: Exploring Accomplishment and Satisfaction //Archives of Rehabilitation Research and Clinical Translation. 2019. Vol. 1. Issues 3-4. Retrieved from: http://www.sciencedirect.com/.

8. Emerson E., Fortune N., Llewellyn G., Stancliffe R. Loneliness, social support, social isolation and wellbeing among working age adults with and without disability: Cross-sectional study//Disability and Health Journal, 2021.Vol. 14. Issue 1. https://doi.org/10.1016/j.dhjo.2020.100965.

9. Trani J.F., Moodley J., Anand P. Graham L., Maw M. T. T. Stigma of persons with disabilities in South Africa: Uncovering pathways from discrimination to depression and low self-esteem. Social Science \& Medicine, 2020. Vol. 265. Retrieved from: http://www.sciencedirect.com/ https://doi.org/10.1016/j.socscimed.2020.113449. 


\section{THE SPECIFICS OF ADAPTATION AND INTERNAL DISORDER PATTERN OF ADULTS WITH DISABILITIES}

This work was supported by the Russian Foundation for Humanities (project №

19-013-00768A)

Adeeva T. N., Tichonova I. V. (Kostroma State University, Kostroma, Russia)

The internal disorder pattern is considered as a component of the Self-concept and is understood as a complex of sensations, knowledge, experiences, and motivational changes associated with the disorder. This phenomenon includes four components: physical, cognitive, emotional, and motivational.

The features of the internal disorder pattern of adults with disabilities (IPD) are defined in the study. The phenomenon of IPD is considered as a factor in the formation of the Self-concept, which means that it can be associated with a person's attitude to himself, to others, with the motives of activity, with the formation of various personality traits. The components of IPD have different contents and severity depending on the variant of dysontogenesis. The greatest severity of the components of IPD is observed in groups of adults with impaired functions of the musculoskeletal system and hearing disorders.

The internal pattern of the disorder is important for the socio-psychological adaptation of the individual. The study determined the relationship of IPD with such indicators of adaptation as emotional comfort, external and internal control, dominance, and avoidance of problems. Some of the IDP parameters are predictors of adaptation parameters for various variants of impaired development.

Keywords: internal disorder pattern, dysontogenesis, adaptation, visual impairment, speech disorders, hearing impairment, mobility disabilities. 